\title{
Post-operative budesonide irrigations for patients with polyposis: a blinded, randomized controlled trial*
}

\author{
Rounak B. Rawal', Allison M. Deal'2, Charles S. Ebert, Jr'1, Vishal H. Dhandha', \\ Candace A. Mitchell', Anna X. Hang', Mitchell R. Gore', Brent A. Senior ',2, \\ Adam M. Zanation ${ }^{1,2}$
}

Department of Otolaryngology - Head and Neck Surgery, University of North Carolina at Chapel Hill, Chapel Hill, NC, USA ${ }^{2}$ Comprehensive Cancer Center, University of North Carolina at Chapel Hill, Chapel Hill, NC, USA
Rhinology 53: 227-234, 2015

DOl:10.4193/Rhino14.196

*Received for publication:

August 16, 2014

Accepted: February 5, 2015

\begin{abstract}
Objective: To compare normal saline (NS) vs. NS + budesonide irrigations in post- functional endoscopic sinus surgery (FESS) patients with chronic rhinosinusitis with polyposis (CRSwNP). Currently, no evidence exists for NS + budesonide irrigation over NS irrigation alone.
\end{abstract}

Study design: Prospective, single-blind, randomized controlled trial.

Methods: Subjects were prospectively enrolled to NS or NS + budesonide arms. Patients were evaluated at pre-operative and three post-operative visits (POV): POV1 (1-2 weeks post-op), POV2 (3-8 weeks post-op), and POV3 (3-6 months post-op). Patients were evaluated by three quality of life (QOL) questionnaires (SNOT-22, RSOM-31, and RSDI) and two olfaction scores (UPSIT and the PEA test).

Results: Fifty patients were randomized, with 25 patients in the NS arm and 25 patients in the NS + budesonide arm. Two patients had unexpected pathology and were excluded from the study. By POV2 and POV3, patients experienced a significant improvement in all three QOL surveys, although the degree of improvement between arms was not significant up through POV3. Neither arm experienced significant olfactory improvement up through POV3.

Conclusions: While both NS and NS + budesonide treatments improve QOL for post-FESS patients, neither intervention significantly increases QOL as compared to the other. Olfaction was not significantly improved in either treatment group.

Key words: Polyposis, sinusitis, chronic rhinosinusitis, budesonide, topical steroid, saline irrigation

\section{Introduction}

Chronic rhinosinusitis (CRS) is a complex, heterogeneous disease process, affecting up to 11.1 million Americans every year ${ }^{(1)}$. CRS is frequently classified by the absence or presence of nasal polyps. Chronic rhinosinusitis with nasal polyposis (CRSwNP) is initially treated medically, but when refractory to maximal medical management, it is treated with functional endoscopic sinus surgery (FESS) followed by medical management to prevent future recurrence. Many post-operative medical therapy regimens currently exist. Often medical management will include saline irrigations, widely thought to improve symptoms by increasing mucociliary transport and removing retained mucus, particulate debris, biofilms, and environmental pathogens ${ }^{(2,3)}$. These have been thought to be more efficacious than saline sprays due to greater volume and pressure and the resultant greater mechanical debridement ${ }^{(4)}$.

Topical steroids may be added to these saline irrigations as a 
means to suppress production of proinflammatory products without the negative systemic effects of chronic oral steroid use. Currently no Level II or greater evidence exists for the use of a normal saline plus topical steroid irrigation in the management of post-surgical patients with CRSWNP ${ }^{(5)}$. The objective of this study is to compare the objective and subjective results of normal saline vs. normal saline plus budesonide irrigations in the post-surgical patient with CRSwNP.

\section{Materials and methods}

Approval for this study was obtained from the University of North Carolina Biomedical Institutional Review Board. All consecutive patients undergoing functional endoscopic sinus surgery for CRS with nasal polyposis were assessed for eligibility at the University of North Carolina at Chapel Hill from August 2011 to March 2012. Guidelines from the CONSORT 2010 statement on guidelines for reporting parallel group randomized trials were used and enrollment methods can be visualized in a resultant flow diagram (Figure 1$)^{(6,7)}$. Inclusion criteria required subjects to be English-speaking patients with CRSwNP who were having functional endoscopic sinus surgery after failing medical management. Patient randomization was allocated by a computerized random number generator; patients were assigned to receive either post-operative normal saline irrigations or normal saline plus budesonide nasal irrigations. This was done prior to trial enrollment with the patient, which was done at the time of his or her pre-operative visit. Demographic data such as age, gender, race, primary co-morbidity (allergic fungal sinusitis, Samter's Triad, cystic fibrosis, or none), secondary comorbidities (diabetes, asthma, hypertension, etc), use of Flonase (GlaxoSmithKline, Brentford, Middlesex, United Kingdom), use of peri-operative prednisone, use of immunotherapy, and compliance rates were all recorded. Lund-MacKay scores were collected for all patients and compared between arms to ensure consistent severity of disease. Peri-operative prednisone regimens consisted of a taper beginning at $40 \mathrm{mg}$, then decreasing to 30 $\mathrm{mg}, 20 \mathrm{mg}$, and $10 \mathrm{mg}$, all at three days each. Patient specimens from surgery were sent to pathology to reconfirm a diagnosis of CRSWNP.

A priori power analysis was done to determine the number of patients required in each arm of the study. Multiple methods to determine the minimal clinically important difference (MCID) for our study were considered. MCID for the SNOT-22 is validated at 8.9 points, but the original study used for validation only studied patients with a 3-month follow-up ${ }^{(8)}$. The SNOT-22 is an outcomes measurement originally extrapolated from the SNOT-20. Unlike the MCID for the SNOT-22, the MCID for the SNOT-20 is validated for patients for the duration of our study (six months) at 0.8 points per question (determined by summation and averaging over all questions) ${ }^{(9)}$. We therefore used the MCID for

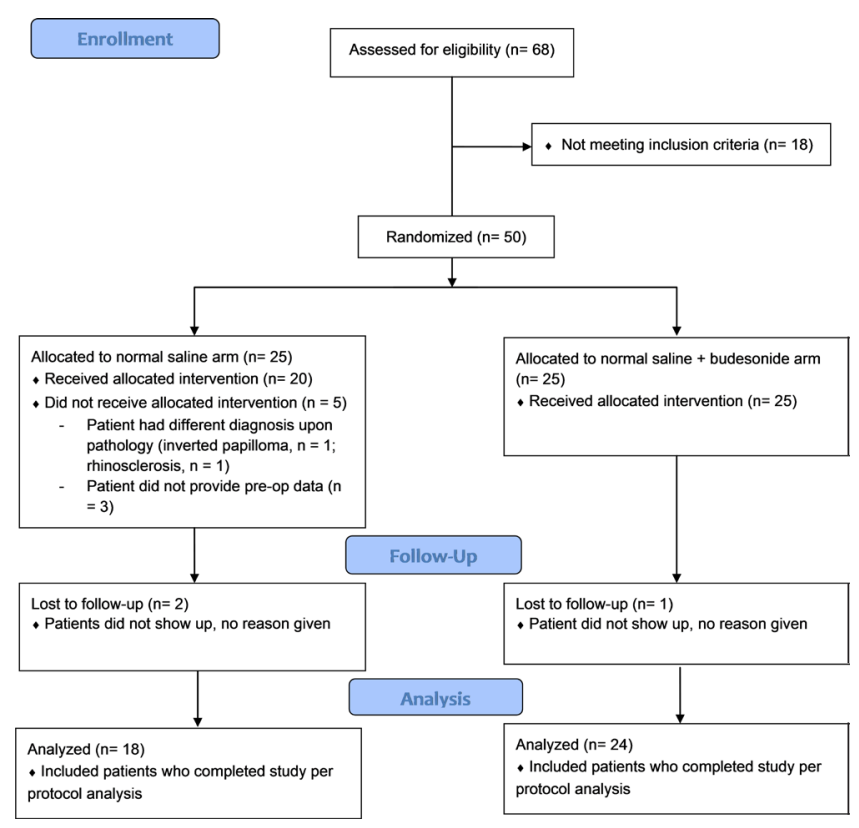

Figure 1. Patient enrollment flow diagram.

the SNOT-20, and extrapolated this to an 18 point MCID in order to use the SNOT-22 for the patients in our study for a follow-up of six months. Using an $\alpha=0.05$, and $\beta=0.8$, eighteen patients were required to be in each arm for a two-treatment parallel design using SNOT-22 as the primary outcomes measure. Considering a 35\% loss to follow-up and non-compliance for treatment, we determined that 25 people were sufficient to enroll in each arm to retain enough patient data for a significant study. Subjects were studied at the pre-operative visit and three postoperative visits (POV). The first POV was conducted in the first two weeks after surgery. POV2 was conducted three to eight weeks after surgery. POV3 was conducted three to six months after surgery. Patients were evaluated for compliance to postoperative instructions at each visit, and if there was any deviation from the irrigation instructions, the patient was recorded as "not compliant".

\section{Irrigations}

Subjects were given instructions on post-operative care. A normal saline solution reserve was created by the patients by mixing the following ingredients: 1.5 teaspoons of canning/pickling salt, 1.5 teaspoons of baking soda, and 1 quart of distilled water. If patients were randomized to the normal saline plus budesonide arm, they were instructed to mix $2 \mathrm{~mL}$ of $0.5 \mathrm{mg} / 2$ $\mathrm{mL}$ budesonide (Pulmicort Respules, AstraZeneca Canada, Mississauga, ON, Canada) into their normal saline solution reserves. Patients were then instructed to irrigate with $60 \mathrm{~mL}$ per nostril twice-daily irrigation for a total of $240 \mathrm{~mL}$ using a high -volume low -pressure irrigating device (Sinus Rinse, NeilMed Pharmaceuticals, Inc, Santa Rosa, CA, USA). 


\section{Outcomes measurements}

Both subjective and objective measurements were made to evaluate efficacy of treatment. Subjective measurements included three quality of life surveys: the Sino-Nasal Outcomes Test (SNOT-22), the Rhinosinusitis Outcomes Measurement Test (RSOM - 31), and the Rhinosinusitis Disability Index (RSDI). Subscores for the RSOM-31 and the RSDI were both recorded. These subjective measurements were made at the pre-operative visit and each of the three post-operative visits.

Objective measurements were made via olfaction testing. OIfaction was tested using two methods: the Phenyl Ethyl Alcohol (PEA) threshold test and the University of Pennsylvania Smell Identification Test (UPSIT). The PEA threshold test is a noninvasive measure, consisting of a single staircase detection threshold paradigm of PEA ranging from full strength to $-6 \log 10$ in half log units ${ }^{(10)}$. PEA was presented in a dual choice model along with a placebo and thresholds were recorded at the level that subjects were able to correctly identify the odorant vial on two consecutive attempts, as the serial dilutions were increased in concentration. The UPSIT consists of four test booklets, each containing 10 pages, with a microencapsulated odorant embedded in a strip in a scratch and sniff fashion ${ }^{(11)}$. Release of the odorant was activated by a led pencil on each page, and a multiple-choice question was posed in a forced-choice format (i.e. if no smell was perceived, a response was still required). Olfactory measurements were made at the pre-operative visit, POV2, and POV3. All efforts were made to collect olfaction testing through each POV, although this was not always feasible.

\section{Blinding}

Patients were enrolled from the clinics of four senior authors (CSE, BAS, MRG and AMZ). Patients were informed of the possibility of entering either treatment arm prior to consenting for the study, and were informed of their study arm placement only after consent was signed. The senior authors blinded as to which arm their patients would be enrolled.

\section{Statistical analysis}

Non-parametric tests were used due to the small sample size per group. Wilcoxon Rank Sums were used for continuous variables and Fisher's Exact tests were used for categorical variables to compare patient and clinical demographics between treatment groups. For quality of life and UPSIT statistics, a change in quality of life or UPSIT score was measured between the pre-operative visit and POV1, POV2, and POV3, respectively. For each change for each treatment, a Wilcoxon Signed Rank test was used to see if the change between the two time points was significantly different from 0 . Then for each change, a Wilcoxon Rank Sum test was used to see if the change between the two time points were significantly different between the two treatment groups. PEA scores were categorized at POV2 and POV3 in relation to the pre-operative visit as improved, same, or worsened. The percentage of people in each of these categories was compared between the two treatment groups using Fisher's Exact testing.

\section{Results}

\section{Study population}

Sixty-eight patients were initially assessed for eligibility, and after excluding those that did not fulfill inclusion criteria, fifty patients were randomized into the study. Twenty-five patients were allocated to the normal saline arm and twenty-five patients were allocated to the normal saline plus budesonide arm. In accordance to CONSORT guidelines, patients were randomized and allocated to treatment arms prior to collection of pre-operative data collection or confirmation of pathology. This resulted in five patients who did not receive the allocated intervention, all in the normal saline arm. Two patients were found with different diagnoses after pathology (inverted papilloma and rhinosclerosis, respectively) and three patients did not provide all of the pre-op data; these five patients therefore were allocated in the study but did not receive intervention. This resulted in a total of twenty patients receiving intervention in the normal saline arm and twenty-five patients receiving intervention in the normal saline plus budesonide arm. Two patients were lost to follow-up in the normal saline arm and one patient was lost to follow-up in the normal saline plus budesonide arm. None of the patients discontinued their interventions, and thus were considered "compliant" during their post-operative visits. Demographic data included a mean and standard deviation of found to be $47 \pm 16$. The most common primary co-morbidity was "none", while the most common secondary co-morbidity was diabetes mellitus. Lund-MacKay scores were collected, with a resultant mean \pm standard deviation of $13.7 \pm 3.9$ for

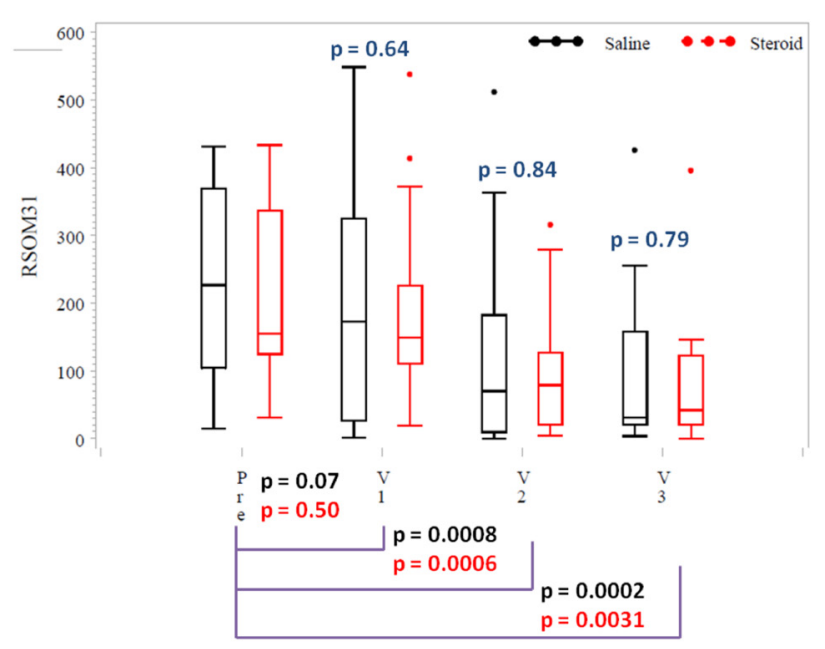

Figure 2. Rhinosinusitis Outcomes Measurement scores. RSOM31= Rhinosinusitis Outcomes Measurement, Pre = Pre-operative visit, V1 = Postoperative Visit 1, V2 = Post-operative Visit 2, V3 = Post-operative Visit 3. 


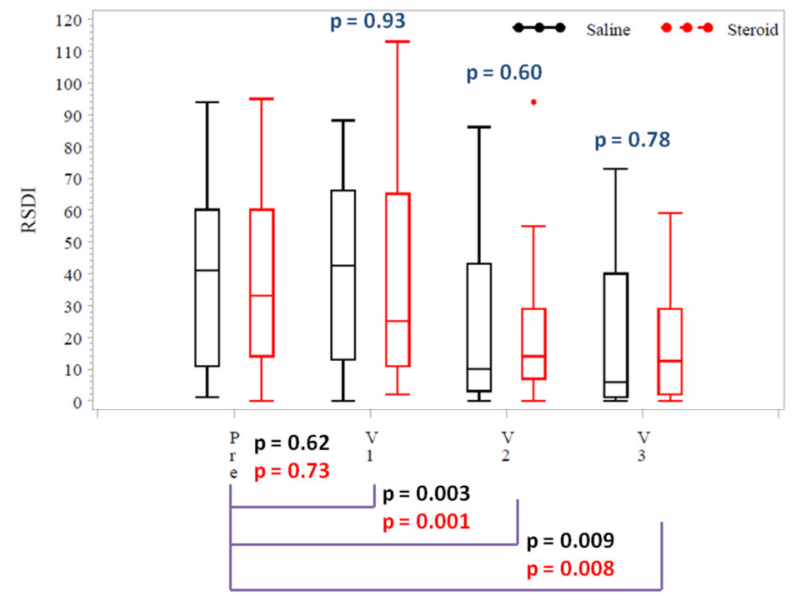

Figure 3. Rhinosinusitis Disability Index scores. RSDI = Rhinosinusitis Disability Index, Pre = Pre-operative visit, V1 = Post-operative Visit 1, V2 $=$ Post-operative Visit 2, V3 = Post-operative Visit 3.

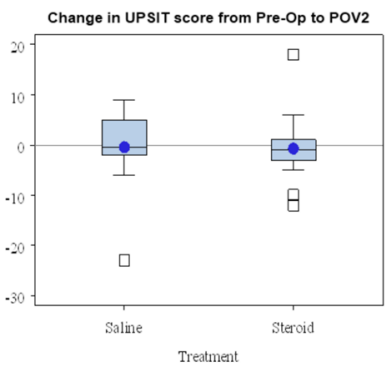

Normal saline (NS): $p=0.80$

NS + Budesonide: $p=0.37$

Between arms: $p=0.55$

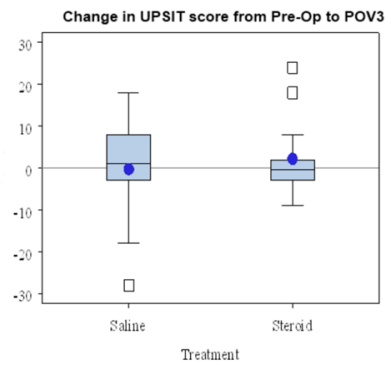

Normal saline (NS): $p=0.88$ NS + Budesonide: $p=0.85$

Between arms: $p=0.99$

Figure 5. University of Pennsylvania Smell Identification Test scores UPSIT = University of Pennsylvania Smell Identification Test, NS = normal saline, Pre - op = Pre-operative visit, POV2 = Post-operative Visit 2, POV3 $=$ Post-operative Visit 3 .

the normal saline arm and $13.8 \pm 4.9$ for the normal saline plus budesonide arm. No statistically significant difference was noted across any demographic when comparing patients from both arms, including use of Flonase, peri-operative prednisone, or immunotherapy, which corresponds to appropriate randomization with well-controlled variables (Table 1).

\section{Quality of life outcomes}

The RSOM31 showed statistically significant differences between pre-operative and post-operative scores by POV2 and POV3 for both normal saline ( $p=0.0008, p=0.0002)$ and normal saline plus budesonide arms $(p=0.0006, p=0.0031)$. There was no statistically significant difference between the arms at any of the three post-operative visits (Figure 2).

The RSDI showed statistically significant differences between

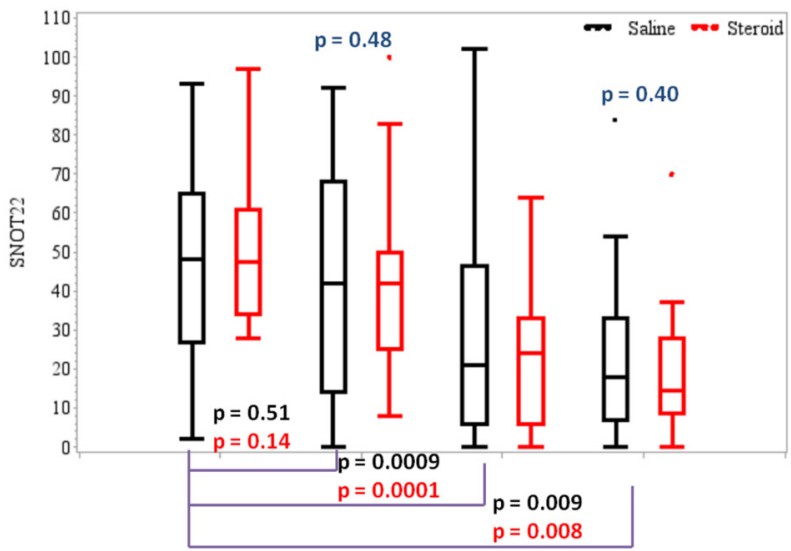

Figure 4. Sinonasal Outcomes Test Scores . SNOT22= Sinonasal Outcomes Test, Pre = Pre-operative visit, V1 = Post-operative Visit 1, V2 = Post-operative Visit 2, V3 = Post-operative Visit 3.
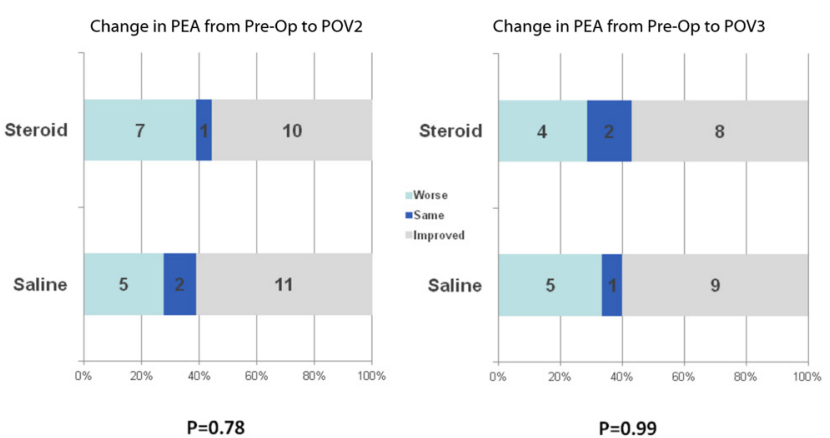

Figure 6. Phenylethylalcohol Threshold Test scores. Pre - op = Preoperative visit, POV2 = Post-operative Visit 2, POV3 = Post-operative Visit 3.

pre-operative and post-operative scores by POV2 and POV3 for both normal saline $(p=0.003, p=0.009)$ and normal saline plus budesonide arms $(p=0.001, p=0.008)$. There was no statistically significant difference between the arms at any of the three post-operative visits (Figure 3).

The SNOT22 showed statistically significant differences between pre-operative and post-operative scores by POV 2 and POV 3 for both normal saline $(p=0.0009, p=0.009)$ and normal saline plus budesonide arms $(p=0.0001, p=0.008)$. There was no statistically significant difference between the arms at any of the three post-operative visits (Figure 4).

\section{Olfactory outcomes}

Differences in UPSIT scores were noted between the preoperative visit and POV2 and the pre-operative visit and POV3. 
Table 1. Patient demographics.

\begin{tabular}{|c|c|c|c|c|c|}
\hline Demographic & Level & All & Saline $(n=23)$ & Steroids $(\mathbf{n}=\mathbf{2 5}$ ) & p-value \\
\hline Age & Median (IQR) & $46.5(37.5,61)$ & $46(35,62)$ & $48(39,57)$ & 0.98 \\
\hline \multirow[t]{2}{*}{ Gender } & Male & $21(44 \%)$ & $12(52 \%)$ & $9(36 \%)$ & \multirow{2}{*}{0.38} \\
\hline & Female & $27(56 \%)$ & 11 (48\%) & $16(64 \%)$ & \\
\hline \multirow[t]{4}{*}{ Race } & White & 39 (81\%) & $16(70 \%)$ & $23(92 \%)$ & \multirow{4}{*}{0.14} \\
\hline & Black & $6(13 \%)$ & $4(17 \%)$ & $2(8 \%)$ & \\
\hline & Hispanic & $2(4 \%)$ & $2(9 \%)$ & 0 & \\
\hline & Other & $1(2 \%)$ & $1(4 \%)$ & 0 & \\
\hline \multirow[t]{4}{*}{ Primary co-morbidity } & None & $33(69 \%)$ & $14(61 \%)$ & $19(76 \%)$ & \multirow{4}{*}{0.43} \\
\hline & Allergic fungal sinusitis & $8(17 \%)$ & $4(17 \%)$ & $4(16 \%)$ & \\
\hline & Cystic Fibrosis & $5(10 \%)$ & $3(13 \%)$ & $2(8 \%)$ & \\
\hline & Samter's Triad & $2(4 \%)$ & $2(9 \%)$ & 0 & \\
\hline \multirow[t]{2}{*}{ Diabetes } & No & $44(92 \%)$ & $22(96 \%)$ & $22(88 \%)$ & \multirow{2}{*}{0.61} \\
\hline & Yes & $4(8 \%)$ & $1(4 \%)$ & $3(12 \%)$ & \\
\hline \multirow[t]{2}{*}{ Hypertension } & No & $36(75 \%)$ & $17(74 \%)$ & $19(76 \%)$ & \multirow{2}{*}{0.99} \\
\hline & Yes & $12(25 \%)$ & $6(26 \%)$ & $6(24 \%)$ & \\
\hline \multirow[t]{2}{*}{ Asthma } & No & $30(63 \%)$ & $16(70 \%)$ & $14(56 \%)$ & \multirow{2}{*}{0.38} \\
\hline & Yes & $18(38 \%)$ & $7(30 \%)$ & $11(44 \%)$ & \\
\hline \multirow[t]{3}{*}{ COPD } & No & 47 (98\%) & $23(100 \%)$ & $24(96 \%)$ & \multirow{3}{*}{0.99} \\
\hline & & & & & \\
\hline & Yes & $1(2 \%)$ & $0(0 \%)$ & $1(4 \%)$ & \\
\hline HIV & No & $48(100 \%)$ & $23(100 \%)$ & $25(100 \%)$ & \\
\hline \multirow[t]{2}{*}{ Use of Flonase } & No & $6(13 \%)$ & $2(9 \%)$ & $4(17 \%)$ & \multirow[b]{2}{*}{0.67} \\
\hline & Yes & 40 (87\%) & 21 (91\%) & 19 (83\%) & \\
\hline \multirow[t]{2}{*}{ Peri-Op Prednisone } & No & $24(50 \%)$ & $10(43 \%)$ & $14(56 \%)$ & \multirow[b]{2}{*}{0.56} \\
\hline & Yes & 24 (50\%) & 13 (57\%) & 11 (44\%) & \\
\hline \multirow[t]{2}{*}{ Immunotherapy } & No & 44 (92\%) & 22 (96\%) & $22(88 \%)$ & \multirow[b]{2}{*}{0.61} \\
\hline & Yes & $4(8 \%)$ & $1(4 \%)$ & $3(12 \%)$ & \\
\hline
\end{tabular}

$\mathrm{IQR}=$ interquartile range. $\mathrm{COPD}=$ chronic obstructive pulmonary disease. Peri-Op $=$ peri-operative

No significant difference in UPSIT score was noted by POV2 or POV3 for neither the normal saline $(p=0.8047, p=0.8804)$ nor the normal saline plus budesonide arms $(p=0.3659, p=0.8523)$. Neither arm differed significantly from each other at either POV2 or POV3 (Figure 5).
PEA thresholds were reported as "Worse", "Same", or "Improved" at POV2 and POV3 as compared to the pre-operative visit. There was no significant difference between normal saline and the normal saline plus budesonide arm by POV2 $(p=0.78)$ and POV3 ( $p=0.99$ ) (Figure 6). 


\section{Discussion}

Our results indicate that following functional endoscopic sinus surgery for patients with CRSwNP, disease specific quality of life is improved with both normal saline irrigations and normal saline plus budesonide irrigations; however, there is no significant difference in the degree of improvement in the short or longterm period between the two. In addition, these patients did not have objective improvement in olfaction using either normal saline irrigations or normal saline plus budesonide irrigations.

Although intranasal saline irrigation with admixed topical steroid has been proven to be safe in a number of trials, the evidence for its use over normal saline irrigations is lacking ${ }^{(12-14)}$. Rotenberg et al. have published the only other current randomized controlled trial comparing these two treatments, but this trial suffers from a lack of broad generalizability since inclusion criteria included only CRSwNP with Samter's Triad, there was a low volume $60 \mathrm{~mL}$ of irrigation delivered, and surgery sometimes only included polypectomy ${ }^{(15)}$. Regardless, Rotenberg et al. also found no significant difference between normal saline and normal saline plus budesonide irrigations in quality of life, radiographic, and endoscopic scores. Jang et al. retrospectively studied a post-operative population of patients who utilized budesonide irrigations and used them as their own controls to compare when they were non-compliant with therapy ${ }^{(16)}$. The authors note that patients had significantly better quality of life SNOT-20 scores but no significant change in endoscopic Lund-Kennedy scores. However, these patients were not using normal saline irrigations when they were non-compliant, possibly masking the stronger effect of irrigant itself rather than the type of irrigant.

A previous Cochrane review has concluded that the beneficial effects of saline irrigations outweigh the drawbacks for the majority of patients ${ }^{(3)}$. There is substantial experimental data to support this approach in the post-surgical setting, including a study by Grobler et al. that showed sinuses of post-operative patients had significantly larger minimal ostial dimensions and greater penetration of irrigation than those of non-operated patients ${ }^{(17)}$. A comprehensive review by Wei et al. in 2013 found that there is a high level of evidence supporting nasal saline in the post-FESS patient for treatment of symptoms of CRSWNP, but that same review found insufficient evidence for use of budesonide irrigations ${ }^{(5)}$. We believe that our observations add to this lack of evidence by providing Level 1B evidence that budesonide irrigations may not offer a significant improvement in patient quality of life or olfaction as compared to normal saline irrigations alone $^{(18)}$.

Budesonide irrigations, while helpful in theory, may not have caused a significantly greater symptomatic change in our patient population for several reasons. The most likely reason may be that the effect of budesonide is masked by the positive effects of irrigant. Irrigations have been shown to increase mucociliary transport and remove mucus, particular debris, biofilms, and environmental pathogens, and their effects may have overcome any added positive benefit of budesonide ${ }^{(2,3)}$. Other possible reasons may include insufficient treatment of nasal mucosa, either through decreased content or insufficient delivery. Higher doses of budesonide may be required to see greater change, although experiments with higher concentrations of budesonide will have to be tempered with concerns for safety and systemic absorption of steroid ${ }^{(12,13)}$. Drug delivery may also have been insufficient, and newer methods for steroid delivery may show promise ${ }^{(19)}$.

Although the data in our study are consistent with previous studies, there are several limitations to the study, which need to be noted. The last post-operative visit recorded in this visit was up to six months post-operatively. Topical nasal steroids may have a lasting impact greater than six months on rates of recurrent surgery required by the patient, and this should be further evaluated. In addition, the PEA threshold tests were studied as categorical variables rather than continuous variables, an inherent limitation to the test. Based on the UPSIT score outcomes, however, we do not believe this necessary limited the strength of our observations. This study also does not assess the potential impact of direct steroid droplets, steroid sprays, or other nonirrigation steroid methods on CRSwNP, although these interventions have been extensively studied by others ${ }^{(5,20-34)}$.

As noted earlier, guidelines from the CONSORT 2010 statement for reporting parallel group randomized trials were used, including using a per-protocol method of statistical analysis. As discussed by others, intention-to-treat and per-protocol analyses both have implicit biases ${ }^{(35,36)}$. An intention-to-treat analysis here would have required imputing missing outcomes data through methods such as "last observation carried forward", a method that could have been justified if outcomes could have been indirectly measured, such as mortality results gathered from a registry. However, in accordance to the multiple criticisms of this method, last observation carried forward would have been inappropriate for quality of life and olfaction data and therefore was not used ${ }^{(37-40)}$. The CONSORT guidelines recognizes these implicit biases in RCT analysis and therefore dropped the request for intention-to-treat analysis; the guidelines instead require a clear description of who was included in each analysis, as we have done ${ }^{(7)}$.

To our knowledge this is the first randomized controlled trial comparing these two post-operative medical therapies for allcause CRSWNP and thus future directions include confirmation 
of our results by other institutions with similar protocols.

\section{Conclusions}

No significant difference was noted between the use of normal saline irrigations and normal saline plus budesonide irrigations in the post-operative patient with CRSwNP. This lack of difference was noted upon three quality of life measures and two olfactory measures. By six months after surgery, both groups of patients improved in quality of life, but not in olfaction. In addition, neither group improved significantly more than the other. Although these data need to be validated by others, the evidence for post-surgical nasal irrigations with steroids in the setting of CRSwNP suggests that normal saline should be first line treatment as this may decrease cost and obviate accessibility issues for patients with CRSwNP.

\section{Acknowledgements}

RBR gratefully acknowledges support from the Doris Duke Charitable Foundation to UNC for support of the Doris Duke Clinical Research Fellowship.

\section{Author contributions}

RBR, CSE, BAS, AMZ: Study design and conceptualization, data acquisition, data. analysis and interpretation, manuscript preparation, critical revision. AMD: Data analysis and interpretation, manuscript preparation. VHD, CAM, AXH, MRG: Data acquisition, data analysis and interpretation.

\section{Conflict of interest}

The authors have no other conflicts of interest to disclose.

\section{References}

1. Bhattacharyya N. Functional limitations and workdays lost associated with chronic rhinosinusitis and allergic rhinitis. Am J Rhinol Allergy. 2012; 26: 120-122.

2. Talbot AR, Herr TM, Parsons DS. Mucociliary clearance and buffered hypertonic saline solution. Laryngoscope. 1997; 107: 500-503.

3. Harvey R, Hannan SA, Badia L, Scadding G. Nasal saline irrigations for the symptoms of chronic rhinosinusitis. Cochrane Database Syst Rev 2007: CD006394

4. Pynnonen MA, Mukerji SS, Kim HM, Adams ME, Terrell JE. Nasal saline for chronic sinonasal symptoms: a randomized controlled trial. Arch Otolaryngol Head Neck Surg. 2007; 133: 1115-1120.

5. Wei CC, Adappa ND, Cohen NA. Use of topical nasal therapies in the management of chronic rhinosinusitis. Laryngoscope. 2013: 123: 2347-2359.

6. Schulz KF, Altman DG, Moher D. CONSORT 2010 Statement: updated guidelines for reporting parallel group randomised trials. BMC Med. 2010; 8: 18

7. Moher D, Hopewell S, Schulz KF, et al CONSORT 2010 explanation and elaboration: updated guidelines for reporting parallel group randomised trials. Int J Surg. 2012; 10: 28-55.

8. Hopkins C, Gillett S, Slack R, Lund VJ, Browne JP. Psychometric validity of the 22-item Sinonasal Outcome Test. Clin Otolaryngol. 2009; 34: 447-454.

9. Piccirillo JF, Merritt MG, Jr., Richards ML. Psychometric and clinimetric validity of the 20-Item Sino-Nasal Outcome Test (SNOT20). Otolaryngol Head Neck Surg. 2002; 126: 41-47.

10. Doty RL. Office procedures for quantitative assessment of olfactory function. Am J Rhinol. 2007; 21: 460-473.

11. Doty RL, Shaman P, Kimmelman CP, Dann MS. University of Pennsylvania Smell Identification Test: a rapid quantitative olfactory function test for the clinic.
Laryngoscope. 1984; 94: 176-178.

12. Bhalla RK, Payton K, Wright ED. Safety of budesonide in saline sinonasal irrigations in the management of chronic rhinosinusitis with polyposis: lack of significant adrenal suppression. J Otolaryngol Head Neck Surg. 2008; 37: 821-825.

13. Sachanandani NS, Piccirillo JF, Kramper MA Thawley SE, Vlahiotis A. The effect of nasally administered budesonide respules on adrenal cortex function in patients with chronic rhinosinusitis. Arch Otolaryngol Head Neck Surg. 2009; 135: 303-307.

14. Welch KC, Thaler ER, Doghramji LL, Palmer JN, Chiu AG. The effects of serum and urinary cortisol levels of topical intranasal irrigations with budesonide added to saline in patients with recurrent polyposis after endoscopic sinus surgery. Am J Rhinol Allergy. 2010; 24: 26-28

15. Rotenberg BW, Zhang I, Arra I, Payton KB. Postoperative care for Samter's triad patients undergoing endoscopic sinus surgery: a double-blinded, randomized controlled trial. Laryngoscope. 2011; 121: 27022705.

16. Jang DW, Lachanas VA, Segel J, Kountakis SE. Budesonide nasal irrigations in the postoperative management of chronic rhinosinusitis. Int Forum Allergy Rhinol. 2013; 3: 708-711.

17. Grobler A, Weitzel EK, Buele A, et al. Pre- and postoperative sinus penetration of nasal irrigation. Laryngoscope. 2008; 118: 20782081.

18. Phillips CB, Sackett D, Badenoch D, Straus S, Haynes B, Dawes M, Howick J. Oxford Centre for Evidence-based Medicine Levels of Evidence. March 2009 [cited 2014 August 1]; Available from: http://www. cebm.net/oxford-centre-evidence-basedmedicine-levels-evidence-march-2009/.

19. Thamboo A, Manji J, Szeitz A, et al. The safety and efficacy of short-term budesonide delivered via mucosal atomization device for chronic rhinosinusitis without nasal polyposis. Int Forum Allergy Rhinol. 2014; 4: 397-402.

20. Pinto JM, Elwany S, Baroody FM, Naclerio RM. Effects of saline sprays on symptoms after endoscopic sinus surgery. Am J Rhinol. 2006; 20: 191-196.

21. Dijkstra MD, Ebbens FA, Poublon RM, Fokkens WJ. Fluticasone propionate aqueous nasal spray does not influence the recurrence rate of chronic rhinosinusitis and nasal polyps 1 year after functional endoscopic sinus surgery. Clin Exp Allergy. 2004; 34: 1395-1400.

22. Rowe-Jones JM, Medcalf M, Durham SR, Richards DH, Mackay IS. Functional endoscopic sinus surgery: 5 year follow up and results of a prospective, randomised, stratified, double-blind, placebo controlled study of postoperative fluticasone propionate aqueous nasal spray. Rhinology. 2005; 43: 2-10.

23. Jorissen M, Bachert C. Effect of corticosteroids on wound healing after endoscopic sinus surgery. Rhinology. 2009; 47: 280-286.

24. Stjarne P, Blomgren K, Caye-Thomasen $P$, Salo S, Soderstrom T. The efficacy and safety of once-daily mometasone furoate nasal spray in nasal polyposis: a randomized, double-blind, placebo-controlled study. Acta Otolaryngol. 2006; 126: 606-612.

25. Vendelo Johansen L, Illum P, Kristensen S, et al. The effect of budesonide (Rhinocort) in the treatment of small and medium-sized nasal polyps. Clin Otolaryngol Allied Sci. 1993; 18: 524-527.

26. Lildholdt $\mathrm{T}$, Rundcrantz $\mathrm{H}$, Lindqvist $\mathrm{N}$. Efficacy of topical corticosteroid powder for nasal polyps: a double-blind, placebo-controlled study of budesonide. Clin Otolaryngol Allied Sci. 1995; 20: 26-30.

27. Holmberg K, Juliusson S, Balder B, et al. Fluticasone propionate aqueous nasal spray in the treatment of nasal polyposis. Ann Allergy Asthma Immunol. 1997; 78: 270-276.

28. Lund VJ, Flood J, Sykes AP, and Richards DH. Effect of fluticasone in severe polyposis. 
Arch Otolaryngol Head Neck Surg. 1998; 124: 513-518.

29. Tos M, Svendstrup F, Arndal H, et al. Efficacy of an aqueous and a powder formulation of nasal budesonide compared in patients with nasal polyps. Am J Rhinol. 1998; 12 183-189.

30. Penttila M, Poulsen P, Hollingworth $K$ Holmstrom M. Dose-related efficacy and tolerability of fluticasone propionate nasal drops 400 microg once daily and twice daily in the treatment of bilateral nasal polyposis: a placebo-controlled randomized study in adult patients. Clin Exp Allergy. 2000; 30: 94-102.

31. Keith $\mathrm{P}$, Nieminen J, Hollingworth $\mathrm{K}$, Dolovich J. Efficacy and tolerability of fluticasone propionate nasal drops 400 microgram once daily compared with placebo for the treatment of bilateral polyposis in adults. Clin Exp Allergy. 2000; 30: 1460-1468.

32. Filiaci F, Passali D, Puxeddu R, Schrewelius C. A randomized controlled trial showing efficacy of once daily intranasal budesonide in nasal polyposis. Rhinology. 2000; 38: 185-190.

33. Jankowski R, Schrewelius C, Bonfils $P$, et al. Efficacy and tolerability of budesonide aqueous nasal spray treatment in patients with nasal polyps. Arch Otolaryngol Head Neck Surg. 2001; 127: 447-452.

34. Small CB, Hernandez J, Reyes A, et al. Efficacy and safety of mometasone furoate nasal spray in nasal polyposis. J Allergy Clin Immunol. 2005; 116: 1275-1281.

35. Nagelkerke N, Fidler V, Bernsen R, Borgdorff $M$. Estimating treatment effects in randomized clinical trials in the presence of non-compliance. Stat Med. 2000; 19: 18491864

36. Sheiner LB and Rubin DB. Intention-to-treat analysis and the goals of clinical trials. Clin Pharmacol Ther. 1995; 57: 6-15.

37. Molnar FJ, Hutton B, Fergusson D. Does analysis using "last observation carried forward" introduce bias in dementia research? CMAJ 2008; 179: 751-753.

38. Ware $\mathrm{JH}$. Interpreting incomplete data in studies of diet and weight loss. N Engl J Med. 2003; 348: 2136-2137.

39. Lane P. Handling drop-out in longitudinal clinical trials: a comparison of the LOCF and MMRM approaches. Pharm Stat. 2008; 7: 93-106.

40. Streiner DL. The case of the missing data: methods of dealing with dropouts and other research vagaries. Can J Psychiatry 2002; 47: 68-75.

Rounak B. Rawal, MD

Department of Otolaryngology-

Head and Neck Surgery

University of North Carolina Memo-

rial Hospitals

170 Manning Dr.

Ground Floor Physician Office Buil-

ding, CB\#7070

Chapel Hill, NC 27599

USA

Tel: +1-919-966-3342

Fax: +1-919-966-7941

E-mail: rrawal@unch.unc.edu 